\title{
Biological activities and chemical compositions of slime tracks and crude exopolysaccharides isolated from plasmodia of Physarum polycephalum and Physarella oblonga
}

Tuyen T.M Huynh', Trung V. Phung ${ }^{2}$, Steven L. Stephenson ${ }^{3}$ and Hanh T.M Tran ${ }^{1 *}$

\begin{abstract}
Background: The myxomycetes derive their common name (slime molds) from the multinucleate trophic stage (plasmodium) in the life cycle, which typically produces a noticeable amount of slimy materials, some of which is normally left behind as a "slime track" as the plasmodium migrates over the surface of a particular substrate. The study reported herein apparently represents the first attempt to investigate the chemical composition and biological activities of slime tracks and the exopolysaccharides (EPS) which cover the surface of the plasmodia of Physarum polycephalum and Physarella oblonga.

Results: Chemical analyses indicated that the slime tracks and samples of the EPS consist largely of carbohydrates, proteins and various sulphate groups. Galactose, glucose and rhamnose are the monomers of the cabohydrates present. The slime tracks of both species and the EPS of Phy. oblonga contained rhamnose, but the EPS of Ph. polycephalum had glucose as the major monomer. In term of biological activities, the slime tracks displayed no antimicrobial activity, low anticancer activity and only moderate antioxidant activity. However, EPSs from both species showed remarkable antimicrobial activities, especially toward Candida albicans (zone of inhibition $\geq 20 \mathrm{~mm}$ ). Minimum inhibitory concentrations of this fungus were found to be $2560 \mu \mathrm{g} / \mathrm{mL}$ and $1280 \mu \mathrm{g} / \mathrm{mL}$ for EPS from Phy. oblonga and Ph. polycephalum, respectively. These EPS samples also showed moderate antioxidant activities. However, they both displayed cytotoxicity towards MCF-7 and HepG2 cancer cells. Notably, EPS isolated from the plasmodium of Phy. oblonga inhibited the cell growth of MCF-7 and HepG2 at the half inhibitory concentration (IC50) of 1.22 and 1. $11 \mathrm{mg} / \mathrm{mL}$, respectively.
\end{abstract}

Conclusions: EPS from Ph. polycephalum plasmodium could be a potential source of antifungal compounds, and EPS from Phy. oblonga could be a potential source of anticancer compounds.

Keywords: Candida albicans, HepG2, Mcf-7, Monomer composition, Slime molds

\footnotetext{
* Correspondence: ttmhanh@hcmiu.edu.vn

${ }^{1}$ School of Biotechnology, International University, VNU-HCM, Block 6,

LinhTrung Ward, Thu Duc District, Ho Chi Minh City 70000, Vietnam

Full list of author information is available at the end of the article
} 


\section{Background}

Exopolysaccharides (EPSs) are macromolecules mainly composed of carbohydrate residues, which are secreted by microorganisms into the surrounding environment. EPSs can serve as centers for bacterial cell aggregation, as nutrient sources and also form a protective barrier for the cell against harsh external conditions [1]. Microbial EPSs have gained a great deal of interest due to their potential biological activities [2]. EPSs isolated from bacteria and fungi have been found to possess inhibitory activities against gram positive and negative bacteria and the $\mathrm{H} 1 \mathrm{~N} 1$ virus [3-5]. EPSs isolated from bacteria and fungi have a significant scavenging ability against superoxide, hydroxyl and DPPH radicals [6-8]. Microbial EPSs also represent a promising source of anticaner agents. Cell-bound galactan exoplysaccharide of Lactobacillus plantarum, at a concentration of $600 \mu \mathrm{g} / \mathrm{mL}$, showed cytotoxic effects of about $56.34 \%$ against the human liver carcinoma (HepG2) cell line [9]. Osama et al. [5] found that EPS isolated from Bacillus marinus showed a strong antitumor property against breast cancer (MCF-7) cell lines and alveolar basal epithelia (A-549) cell lines at concentration of $100 \mu \mathrm{g} / \mathrm{mL}$. In addition, EPS from Aspergillus aculeatus displayed a strong anti-proliferation effect on human cervical carcinoma cells (Hela), human breast carcinoma cells (MCF-7) and gastric carcinoma cells (MGC-803) with inhibition rates of $53.9 \%, 29.1 \%$ and $34.1 \%$, respectively, at a concentration of $1000 \mu \mathrm{g} /$ $\mathrm{mL}$ for $48 \mathrm{~h}$ [10].

The myxomycetes are a group of primitive phagotrophic eukaryotes. The myxomycete life cycle consists of two very different trophic stages-uninucleate amoebae and a distinctive multinucleate structure, the plasmodium. Under favorable conditions, the plasmodium converts into fruiting bodies [11]. Having the characteristics of both fungi and protozoans make the myxomycetes an unusual group of microorganisms. More than 100 secondary metabolites have been isolated from myxomycetes, and many of among those are novel bioactive compounds [12]. In addition to potential antimicrobial compounds such as a new glycerolipid (bahiensol) isolated from the plasmodium of Didymium bahiense [13], stigmasterol and fatty acids obtained from plasmodial extracts of Phy. oblonga [14], some remarkable anticancer compounds from myxomycetes have also been reported. Cyclic phosphatidic acid (CPA), a novel bioactive lipid isolated from $\mathrm{Ph}$. polycephalum was found to have ability to inhibit cancer cell invasion and metastasis [15]. In addition, two new bisindole alkaloids isolated from the fruiting bodies of Lycogala epidendrum showed cytotoxicity against HeLa cells and Jurkat cells with relatively low IC50 values [16]. In similar research, Kehokorins A, a novel dibenzofuran isolated from the fruiting bodies of Trichia favoginea var. persimilis was found to have significantly high cytotoxicity toward HeLa cells with an IC50 value of $1.5 \mu \mathrm{g} / \mathrm{mL}$ [17].

Among the myxomycetes, those members of the Physarales (e.g., Physarum polycephalum) often form large plasmodia and are relatively easy to culture on synthetic media. When cultured in liquid media, microplasmodia are formed instead of plasmodia. Both microplasmodia and plasmodium lack cell walls. On solid media, the plasmodium is a slimy mass of protolasm which is capable of moving around. In the absence of cell walls, the slime sheath represents the only protection from injury and the environment, and material from the slime sheath is left behind as a slime track as the plasmodium migrates over the surface of a given substrate [18]. There have been a few studies of the chemical composition of EPSs isolated from microplasmodia in liquid culture, but there appear to be no studies of the properties of EPS and slime tracks isolated from solid cultures of myxomycete plasmodia. The chemical characteristics of the EPSs seem to strongly depend upon the culture media used. McCormick et al. [19] found that Ph. polycephalum microplasmodial cultures started to produce more EPS when the cells were converted into spherules and reported that the EPS is a sulfated galactose polymer containing trace amounts of rhamnose. Simon and Henney [20] reported that the EPS was a glycoprotein. More recently, Sperl [21] found the EPSs produced by $\mathrm{Ph}$. polycephalum consisted of two galactans with different ratios of phosphorous and sulfur. To the best of our knowledge, there has been only one report on the biological activity of myxomycete EPS, and this was published by Asgari and Henney [22]. Their research found that the EPS secrected by the microplasmodia of Physarum flavicomum in liquid culture was composed mainly of glycoprotein and could inhibit the cell growth and division of Bacillus subtilis.

Given the fact that microbial EPSs have been found to have potential biological activities and myxomycete plasmodia produce a noticeable amount of slimy materials, it seemed worthwhile to evaluate the biological activities (antimicrobial, antioxidant and anticancer activities) and to determine the chemical characteristics of slime tracks and EPS samples isolated from Physarella oblonga and Physarum polycephalum. These two species were chosen because of their sample availability and their ease to culture.

\section{Results and discussion}

\section{EPS production of Phy. oblonga and Ph. polycephalum}

The medium used for cultivation of myxomycete plasmodia was adapted from the research of Henney and Henney [23]. We attempted to replace glucose in the original medium with other carbon sources (e.g., oyster mushroom powder [since the oyster mushroom is one of 
the favorite food sources of some myxomycete plasmodia in the nature], rice bran and galactose). However, preliminary results showed that $\mathrm{Ph}$. polycephalum preferred glucose and Phy. oblonga grew better in water agar without glucose (Phy. oblonga has agar hydrolytic activity). As such, for slime track and EPS production, typical plasmodia of Ph. polycephalum and Phy. oblonga were transferred to nutrient and water agar, respectively, and incubated under dark condition at $25{ }^{\circ} \mathrm{C}$ for 7 days (Fig 1). The amounts of slime track material and EPSs obtained are presented in Table 1 . The amounts of slime track material obtained from both species were higher than those of EPSs still in contact with the plasmodium.

\section{Chemical composition of the slime track and EPS samples from Phy. oblonga and Ph. polycephalum}

The carbohydrate, protein and sulfate contents of EPSs are listed in Table 2. The total carbohydrate content of the samples varied from 55 to $82 \%$ according to the phenol-sulfuric acid method. Sulfated groups and protein made up small proportions (Table 2). In general, the

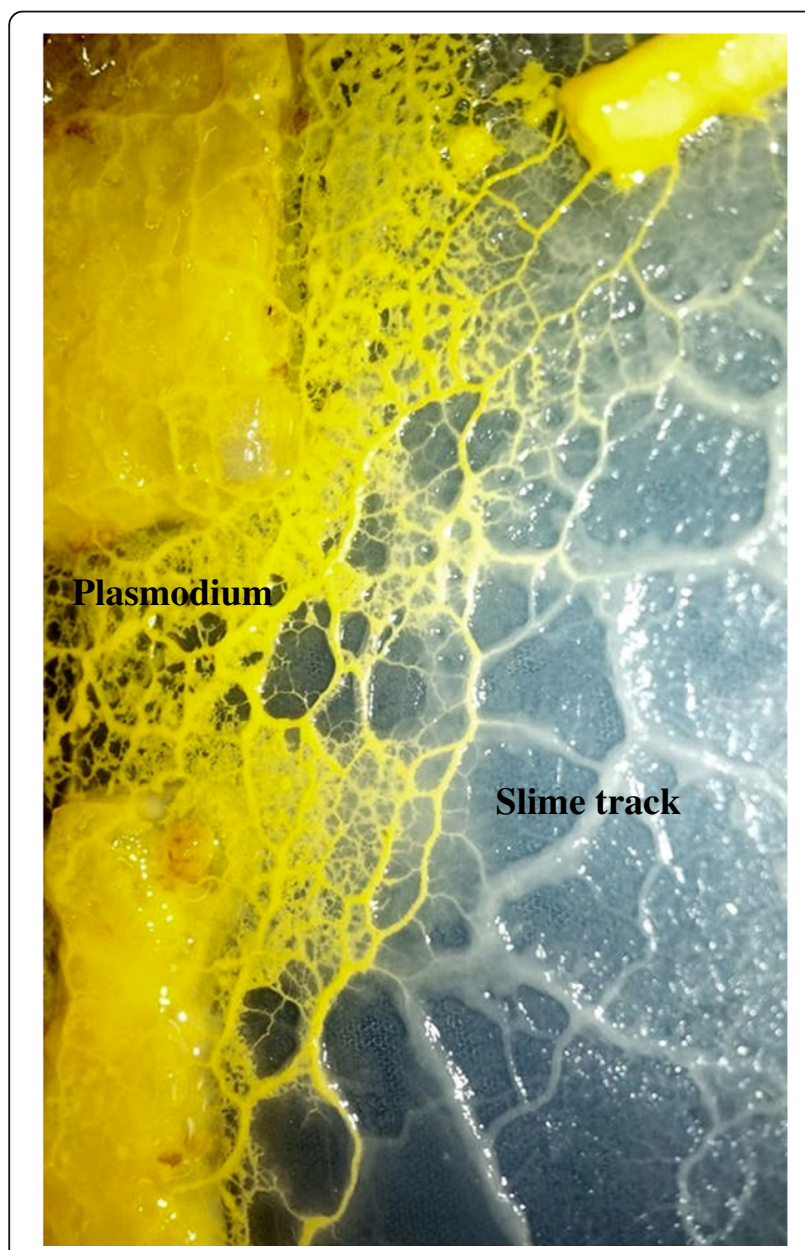

Fig. 1 Plasmodium and slime track
Table 1 Amounts of slime track and EPSs isolated from cultures of Phy. oblonga and Ph. polycephalum

\begin{tabular}{lll}
\hline & Sample & Amount $(\mathrm{g} / \mathrm{L})$ \\
\hline Phy. oblonga & Slime track & $0.51 \pm 0.12$ \\
& EPS & $0.28 \pm 0.09$ \\
P. polycephalum & Slime track & $0.65 \pm 0.10$ \\
& EPS & $0.43 \pm 0.07$ \\
\hline
\end{tabular}

EPS and slime track of Phy. oblonga had greater amounts of carbohydrate compared to those of Ph. polycephalum. However, the samples from the latter species had higher percentages of sulfate content. When comparisons are made between the slime track and EPS samples of each species, the amounts of carbohydrates of the slime tracks were higher than that of the EPS, and this applied for both species.

The slime tracks and EPSs were depolymerized by using the TFA hydrolysis method. The monosaccharide compositions of the EPSs produced by Phy. oblonga and $P$. polycephalum were detected by TLC, and their quantities were measured by GC-FID analysis. The data obtained data are displayed in Fig. 2 and Table 3.

Table 3 showed that the slime track and EPS samples contained glucose, galactose and rhamnose and rhamnose was the major monosaccharide of the EPS from Phy. oblonga and the slime tracks of both species, for which it accounted for $66.37 \%, 62.58 \%$ and $71.46 \%$, respectively. In contrast, EPS from $\mathrm{Ph}$. polycephalum was composed mainly of glucose (50.87\%).

The present study is the first to determine the monomer compositions of EPSs isolated from Phy. oblonga. However, with Ph. polycephalum, the results reported have varied from one study to another. Extracellular slime from broth cultures (containing glucose as the carbon source) of Ph. polycephalum was found to contain galactose, sulfate, and trace amounts of rhamnose [19]. However, Simon and Henny [20] found that slime production of $\mathrm{Ph}$. rigidum, $\mathrm{Ph}$. flavicomun and $\mathrm{Ph}$. polycephalum contained a single sugar component of galactose when cultured on media containing glucose as the carbon source. Similar results for Ph. polycephalum were also reported by Farr et al. [24]. In general, monomer compositions and their ratios in microbial EPSs are influenced by the carbon source in the culture medium $[25,26]$. However, with the myxomycetes, there would appear to be some other factors involved. Ph. polycephalum in our study was cultured on a glucose-based solid medium, but the monomer composition was completely different from what has been reported in other studies. It is possible that plasmodia produce different kinds of slime material as compared to microplasmodia. 
Table 2 Total carbohydrate, protein and sulfate contents of the slime track and EPSS

\begin{tabular}{llll}
\hline Crude EPS & Total carbohydrate (\%) & Protein (\%) & Sulfate content (\%) \\
\hline Phy. oblonga slime track & $82.13 \pm 0.037$ & $7.58 \pm 0.02$ & $1.50 \pm 0.37$ \\
Phy. oblonga EPS & $76.80 \pm 0.052$ & $19.81 \pm 0.02$ & $2.44 \pm 0.05$ \\
Ph. polycephalum slime track & $63.94 \pm 0.056$ & $12.70 \pm 0.05$ & $5.23 \pm 0.04$ \\
Ph. polycephalum EPS & $56.42 \pm 0.061$ & $30.94 \pm 0.04$ & $11.26 \pm 0.02$ \\
\hline
\end{tabular}

\section{Antimicrobial activity of EPSs against pathogens}

Antimicrobial activities of the EPS and slime track samples as determined by the agar diffusion method are presented in Table 4.

The results indicate that there were significant differences in antimicrobial activities among the samples. The slime tracks of both two species did not exhibit any inhibitory activity against the strains of microbes tested. This could be explained by the theory that myxomycete plasmodia leave slime tracks behind when migrating simply to mark the area which has been exploited for food resources [12]. In contrast, isolated EPS from plasmodia showed promising activities towards $S$. aureus and $C$. albicans, whereas $C$. albicans was found to be the most susceptible to the EPSs from both species (zone of inhibition $\geq 20 \mathrm{~mm}$ ) (Table 4). The antimicrobial activities of the EPSs which are still in contact with the plasmodia would be explained by the possibility that these compounds would protect the plasmodia from external factors, including other microorganisms.

The results obtained for antimicrobial activities in the present study agree with those reported in some previous studies relating to the antimicrobial property of microbial EPSs. Asgari and Henney [22] found that the cell growth and division of Bacillus subtilis (a gram positive bacterium) was inhibited by slime secreted by $P h$. flavicomun. The degradation of the cell wall caused morphological changes such as swollen cells or cell lysis. Li et al. [27] found that EPS from Lactobacillus plantarum exhibited inhibitory activities against $S$. aureus and $C$.

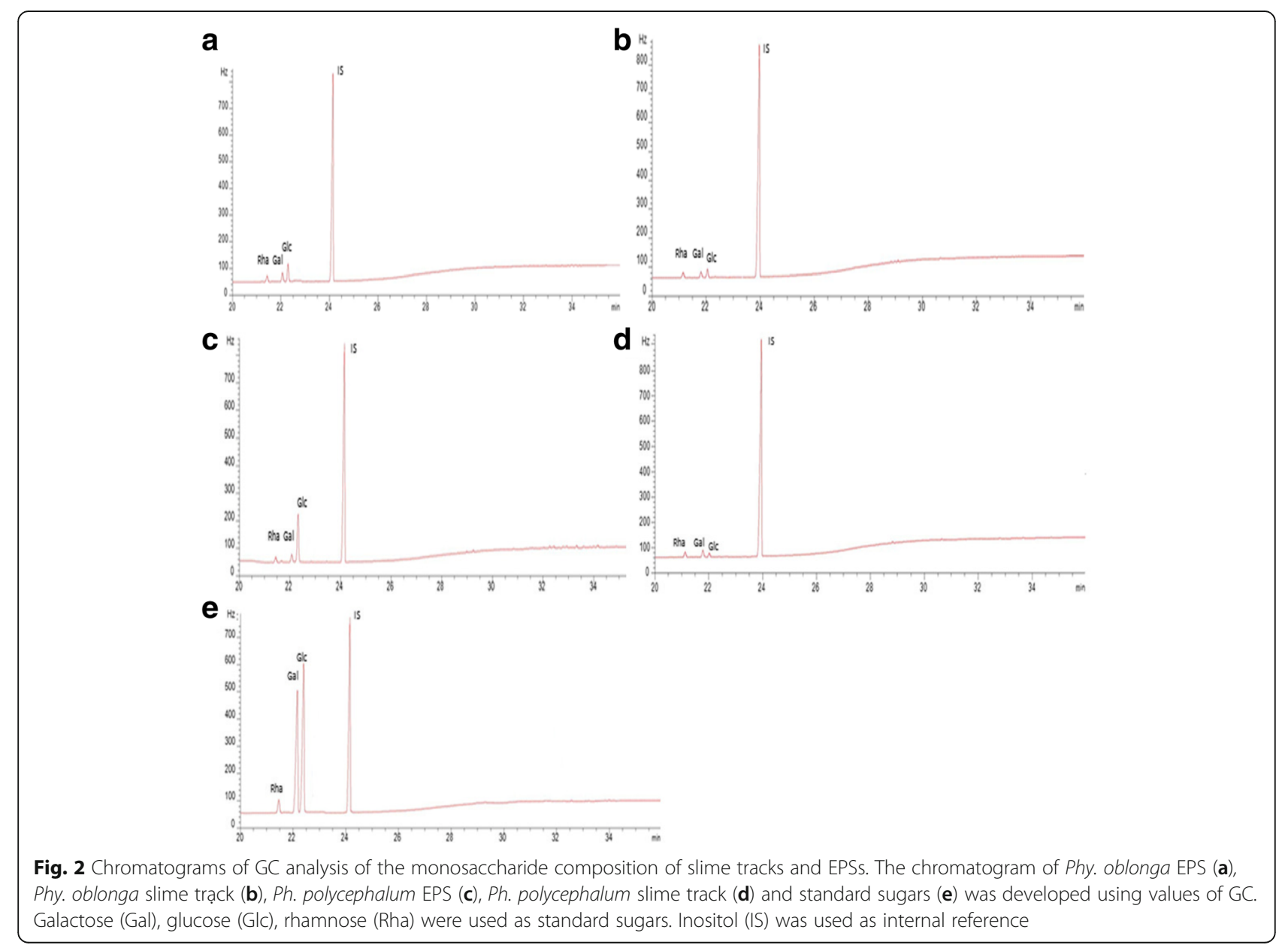


Table 3 Monomer compositions of crude EPSs obtained from cultures of Ph. polycephalum and Phy. oblonga

\begin{tabular}{lllll}
\hline Monomer composition $(\% w / w)$ & POS & POP & PPS & PPP \\
\hline Galactose & 15.65 & 14.09 & 19.43 & 9.47 \\
Glucose & 17.96 & 23.32 & 9.1 & 50.87 \\
Rhamnose & 66.37 & 62.58 & 71.46 & 39.65
\end{tabular}

POS and POP are slime track and EPS samples from Phy. oblonga, whereas PPS and PPP are slime track and EPS samples from $P h$. polycephalum

albicans. EPS from Enterobacter faecalis showed significantly high activity toward C. albicans [28].

The MIC values of the EPS samples from $P h$. polycephalum and Phy. oblonga were studied against $C$. albicans and $S$. aureus. The data obtained are shown in Table 5.

With respect to their ability against $S$. aureus, the MIC value of the $\mathrm{Ph}$. polycephalum EPS was almost the same with that of Phy. oblonga. However, EPS from $\mathrm{Ph}$. polycephalum showed much better antifungal activity, since the MIC value $(1280 \mu \mathrm{g} / \mathrm{mL})$ of the EPS from this species against $C$. albicans was just about a half that from Phy. oblonga $(2560 \mu \mathrm{g} / \mathrm{mL})$ and twice when compared with the standard antifungal drug $(640 \mu \mathrm{g} / \mathrm{mL})$ (Table 5). However, this EPS is not yet purified. Nevertheless, EPS from Ph. polycephalum appears to have the potential for treatment of $C$. albicans. However, it should be noted that the MBC or MFC values are higher than the MIC values. This suggests that the compound would easily inhibit microbial growth at low concentrations, but leading to actual microbial death would require higher doses.

\section{Antioxidant activity}

In this part of our study, in vitro antioxidant activities of the EPS samples with the concentration range of 0-6.0 mg/mL from Phy. oblonga and Ph. polycephalum were determined by DPPH assay and compared with that of ascorbic acid. Figure 3 illustrates that there was not a major difference observed between radical scavenging ability of slime track and EPS extracts from Phy.

Table 4 Antimicrobial activities of EPS and slime track samples from Phy. oblonga and Ph. polycephalum

\begin{tabular}{llllll}
\hline Microorganism & \multicolumn{4}{l}{ Inhibition zone $(\mathrm{mm})$} & \\
\cline { 2 - 6 } & Antibiotics & POS & POP & PPS & PPP \\
\hline B. cereus & $19.75 \pm 0.23$ & - & - & - & - \\
S. aureus & $23.00 \pm 0.41$ & - & $12.75 \pm 0.28$ & - & $10.05 \pm 0.40$ \\
E. coli & $18.50 \pm 0.35$ & - & - & - & - \\
S. typhi & $16.78 \pm 0.52$ & - & - & - & $8.30 \pm 0.12$ \\
C. albicans & $32.00 \pm 0.22$ & - & $20.05 \pm 0.36$ & - & $23.00 \pm 0.28$
\end{tabular}

$D$ diameter of inhibition zone; -: No inhibition observed $(D<10 \mathrm{~mm}$ ); +: $10<\mathrm{D}<15 \mathrm{~mm} ;++$ : $15<\mathrm{D}<20 \mathrm{~mm} ;+++$ : $\mathrm{D}>20 \mathrm{~mm}$

POS and POP are slime track and EPS samples from Phy. oblonga, whereas PPS and PPP are slime track and EPS samples from Ph. polycephalum
Table 5 Minimum inhibitory concentration (MIC) and minimum bactericidal concentration (MBC) or minimum fungicidal concentration (MFC) of EPS and slime track samples from Phy. oblonga and Ph. polycephalum

\begin{tabular}{llll}
\hline & \multicolumn{3}{l}{ MIC (MBC or MFC) $\mu \mathrm{g} / \mathrm{mL}$} \\
\cline { 2 - 4 } Microorganism & POP & PPP & Standard antibiotic $^{\text {a }}$ \\
\hline S. aureus & $5120(5120)$ & $5120(20480)$ & 1280 \\
C. albicans & $2560(10240)$ & $1280(5120)$ & 640 \\
\hline
\end{tabular}

${ }^{a}$ Standard antibiotics include gentamycin (antibacterial drug) and ketoconazole (antifungal drug). POP indicates EPS samples from Phy. oblonga, whereas PPP are EPS samples from Ph. polycephalum

oblonga and Ph. polycephalum at an initial concentration $1.0 \mathrm{mg} / \mathrm{mL}$. However, at the higher sample concentrations, EPS isolated from a plasmodium showed higher radical scavenging ability than EPS isolated from the slime track material in both species. EPS from Phy. oblonga showed maximum DPPH scavenging activity $(80.41 \%)$ at a concentration of $6 \mathrm{mg} / \mathrm{ml}$, whereas that of ascorbic acid was $99.56 \%$.

The EC50 is the concentration of antioxidant needed to obtain a $50 \%$ antioxidant effect, and is typically used as a parameter to express or compare the antioxidant capacity of different compounds. Lower EC50 values show a higher antioxidant activity [28]. EC50 values of the EPS samples and ascorbic acid are displayed in Table 6.

According to the EC50 data, the slime track and EPS samples from Phy. oblonga showed higher scavenging abilities than those from $P h$. polycephalum. These data also indicated that EPSs and slime tracks from $P h$. polycephalum and Phy. oblonga have comparable antioxidant capacity with some common edible mushrooms [29-32]. However, the antioxidant activities these samples were far smaller than ascorbic acid.

\section{In vitro cancer cell line cytotoxicity assays}

In this experiment, crude EPS and slime track samples from Phy. oblonga and Ph. polycephalum were subjected to in vitro cytotoxicity SRB assay with fibroblast and cancer cell lines. Cells were treated with EPSs ranging from 0.25 to $1.5 \mathrm{mg} / \mathrm{mL}$ and incubated for $48 \mathrm{~h}$, and then the cell inhibitory rate was measured by using a spectrophotometer. The data obtained data are shown in Fig. 4.

The results indicate that EPSs possess different levels with respect to their toxicity effects against the cancer cell lines. At low concentrations $(0.25-0.5 \mathrm{mg} / \mathrm{mL})$, none of negatives effect on the proliferation of cancer cell lines were observed. However, EPSs were found to show anti-proliferation when the concentration increased from 0.75 to $1.5 \mathrm{mg} / \mathrm{mL}$.

EPSs isolated from a plasmodium showed higher inhibition rates against the cancer cell lines than EPSs isolated from the slime tracks. Most notably, EPS from Phy. oblonga showed significantly higher inhibitory 


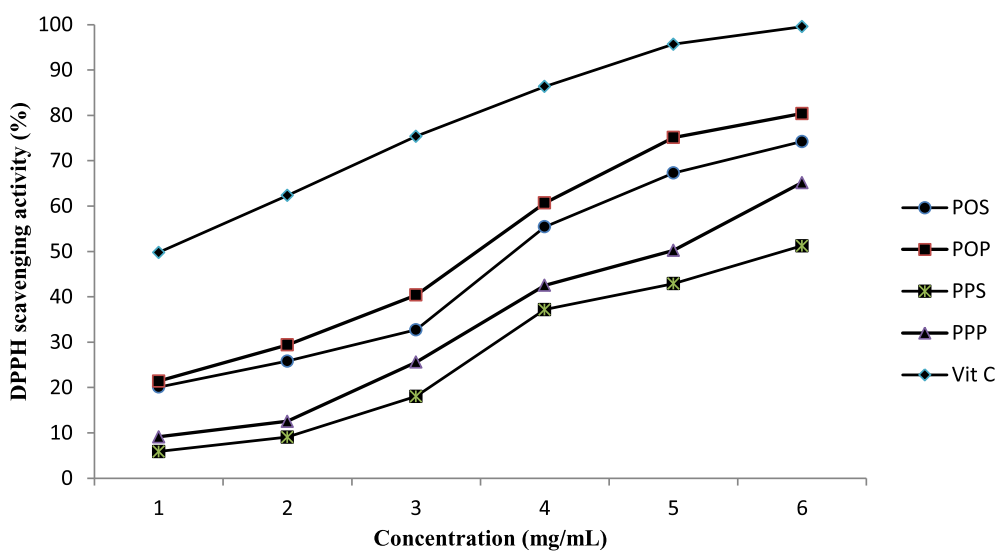

Fig. 3 Antioxidant activities of EPSs of Phy. oblonga and Ph. polycephalum in vitro. POS and POP are slime track and EPS samples from Phy oblonga, whereas PPS and PPP are slime track and EPS samples from Ph. polycephalum

activities against MCF-7 and HepG2 when compared to that of Ph. polycephalum.

The half inhibitory concentrations (IC50) of the EPS sample from Phy. oblonga toward MCF-7 and HepG2 were found as 1.22 and $1.11 \mathrm{mg} / \mathrm{mL}$, respectively. However, these activities are not comparable to the positive control (camptothecin).

Microbial EPS have been found to have antiproliferation effects against HepG2 and MCF-7 cells. Wang et al. [9] reported that at the concentration of $600 \mu \mathrm{g} / \mathrm{ml}$, purified EPS from Lactobacillus plantarum could suppress proliferation of HepG2 cells by $56.34 \%$ when treated for $72 \mathrm{~h}$. In addition, Osama et al. [5] found that (IC50) of purified EPS from Bacillus marinus in the MCF-7 was $118.0 \mu \mathrm{g} / \mathrm{mL}$ after $48 \mathrm{~h}$.

\section{Conclusions}

Culturing myxomycete plasmodia is challenging, but it is possible with the right medium components (e.g., carbon source) selectively used for each species. For example, agar is more suitable for cultivation of Phy. oblonga, but glucose is a better carbon source for Ph. polycephalum.

The slime track and EPS samples from Ph. polycephalum and Phy. oblonga were found to consist of glucose, galactose and rhamnose. Among these, rhamnose was

Table 6 EC50 values of the slime track and EPS samples from Phy. oblonga and Ph. polycephalum

\begin{tabular}{ll}
\hline Sample & EC50 value $(\mathrm{mg} / \mathrm{mL})$ \\
\hline Phy. oblonga slime track & $3.92 \pm 0.22$ \\
Phy. oblonga EPS & $3.59 \pm 0.19$ \\
Ph. polycephalum slime track & $5.99 \pm 0.10$ \\
Ph. polycephalum EPS & $4.87 \pm 0.31$ \\
Ascorbic acid & $0.78 \pm 0.05$ \\
\hline
\end{tabular}

the major monomer of the EPS from Phy. oblonga and the slime tracks from both species, but EPS from Ph. polycephalum contained mainly glucose. This difference may be because of the use of different carbon sources or it could be simply just because of the unique nature of each species. However, since monomer composition is one of the major factors other than molecular weight, structure of the polymeric backbone and degree of branching which decide the biological activities of microbial EPSs. Thus, when one tries to enhance the amount of EPS production by altering medium composition and cultivation condition, the effect those conditions on EPS compositions and subsequently EPS activities should be taken in consideration along with the amount of EPS.

The slime tracks from both two species showed no antimicrobial activity, low anticancer activity, and moderate antioxidant activity. These results support the theory that function of the slime tracks of myxomycetes relates more to marking the area which has been exploited for food resources as the plasmodia migrate from one area to another.

On the other hand, EPS samples from the two species displayed significant inhibitory activities against $C$. albicans and S. aureus, and both of them had anticancer activities against MCF-7 and HepG2. More importantly, EPS from Phy. oblonga was found to have significantly higher inhibitory activities. The IC50 values of this sample on MCF-7 and HepG2 were $1.22 \mathrm{mg} / \mathrm{mL}$ and $1.11 \mathrm{mg} / \mathrm{mL}$, respectively. The differences in biological activities of the slime track and the EPS which is still in contact with the plasmodia suggest that they probably have different functions for the particular species of myxomycetes. EPS purification should be considered in future works to enhance the biological activities.

Myxomycetes are a unique group of microorganisms which could be a potential source of bioactive compounds. 

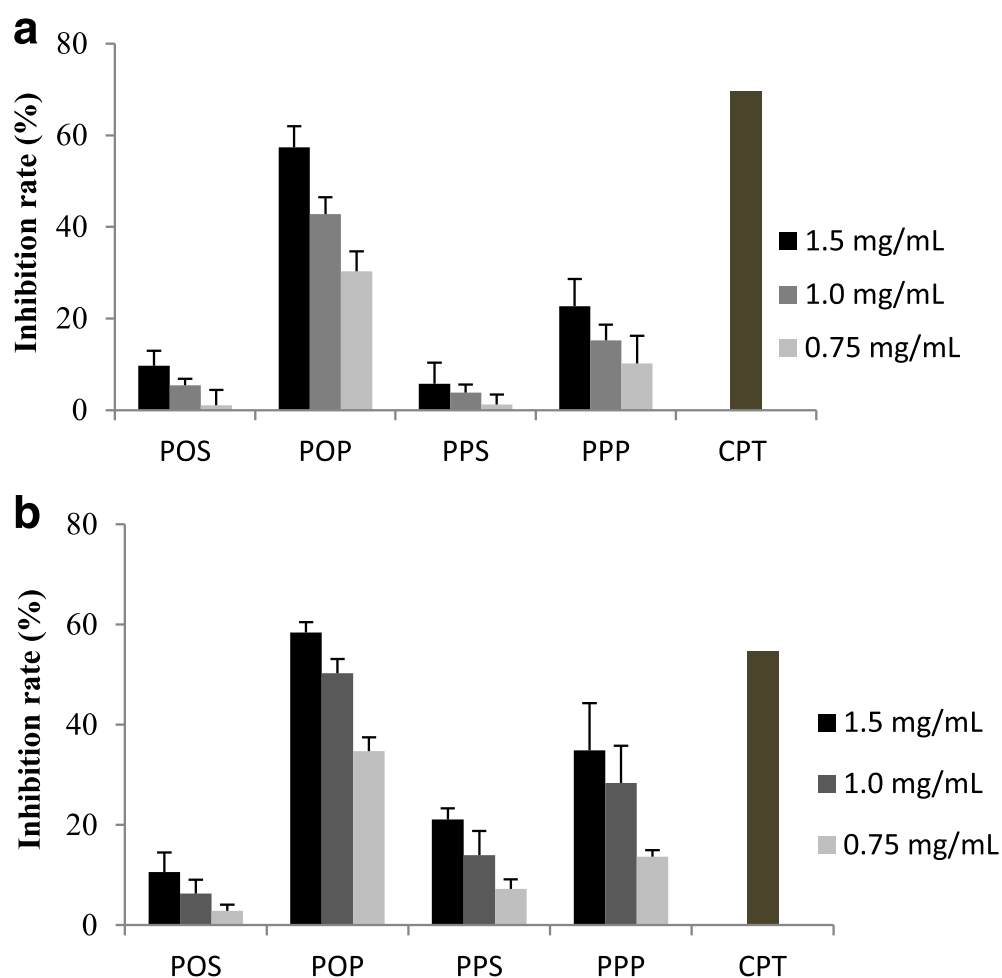

Fig. 4 Growth inhibition of MCF-7 (a) and HepG2 (b) cancer cell lines by treating with crude EPS extracts from Phy. oblonga and Ph. polycephalum in comparison with camptothecin standard (CPT) with the concentration of $0.005 \mu \mathrm{g} / \mathrm{mL}$ via SRB assay. POS, POP, PPS and PPP were represented in the Phy. oblonga slime track, Phy. oblonga EPS, Ph. polycephalum slime track and Ph. polycephalum EPS

\section{Methods}

\section{Materials}

The strain of Ph. polycephalum used in the present study was obtained as a sclerotium from the Carolina Biological Supply Company (Burlington, North Carolina, USA). The Phy. oblonga plasmodium was generated from a fruiting bodies collected from a moist chamber culture prepared from forest floor litter.

Nutrient agar was used for the plasmodial culture of Ph. polycephalum $(1.0 \mathrm{~L}$ of the nutrient agar contained $100 \mathrm{~mL}$ of a basal salt solution, $5.0 \mathrm{~g}$ of glucose, $2.5 \mathrm{~g}$ of yeast extract, $20.0 \mathrm{~g}$ of agar, and $900 \mathrm{~mL}$ of distilled water adjusted to $\mathrm{pH}$ 5.5). The basal salt solution contained $29.78 \mathrm{~g}$ of citric acid, $33.10 \mathrm{~g}$ of $\mathrm{K}_{2} \mathrm{HPO}_{4}$, $2.50 \mathrm{~g}$ of $\mathrm{NaCl}, 1.00 \mathrm{~g}$ of $\mathrm{MgSO}_{4} .7 \mathrm{H} 2 \mathrm{O}, 0.50 \mathrm{~g}$ of $\mathrm{CaCl}_{2} .2 \mathrm{H} 2 \mathrm{O}$, and $1000 \mathrm{~mL}$ distilled water [23, 33].

Water agar was used for the Ph. polycephalum sclerotium and spore germination and plasmodial culture of Phy. oblonga (1.0 L of water agar consisted of $15 \mathrm{~g}$ of agar and $1000 \mathrm{~mL}$ of water).

Pathogenic microorganisms, including Bacillus cereus VTCCB 1005, Escherichia coli JM 109, Samonella typhi ATCC 19430, Staphylococcus aureus ATCC 43300 and Candida albicans ATCC 141 were used. The bacteria were grown on LB agar medium (1.0 L of LB agar containing 10.0 gof $\mathrm{NaCl} ; 5.0 \mathrm{~g}$ of yeast extract; $10.0 \mathrm{~g}$ of peptone; $20.0 \mathrm{~g}$ of agar and $1000 \mathrm{~mL}$ of distilled water adjusted to $\mathrm{pH}$ 7.0) and the fungus was grown on Saboroud agar medium (1.0 L of Saboroud agar containing $40.0 \mathrm{~g}$ of glucose; $10.0 \mathrm{~g}$ of peptone; $20.0 \mathrm{~g}$ of agar and $1000 \mathrm{~mL}$ of distilled water adjusted to $\mathrm{pH}$ 5.5).

Cancer cell lines (breast carcinoma MCF-7 and liver carcinoma HepG2 cells) and fibroblast cells were grown in DMEM $10 \%$ FBS medium and mantained at $37^{\circ} \mathrm{C}$ in a $5 \% \mathrm{CO}_{2}$ incubator.

\section{Plasmodial culture}

Spore gernination, sclerotium activation and inoculum preparations of Ph. polycephalum and Phy. oblonga were carried out following Tran et al. [34, 35]. For plasmodial cultures and EPS production, a small piece of agar a containing actively growing plasmodium covered oatmeal flakes was transferred to a plate containing water agar (for Phy. oblonga) and nutrient agar (for Ph. polycephalum). The plasmodial cultures were incubated in the dark at $25{ }^{\circ} \mathrm{C}$ for 5 days, after which the slime tracks and plasmodia were collected.

\section{Isolation of slime tracks and EPSs from the plasmodial cultures}

Slime tracks were simply scraped off the surfaces of the plasmodial cultures. For EPS isolation, fresh plasmodia 
were carefully collected in $10 \mathrm{~mL}$ of sterile distilled water without discrupting the plasmodium to avoid extracting the intracellular components. The sample was gentely vortexed and centrifuged at $9000 \mathrm{rpm}, 4{ }^{\circ} \mathrm{C}$ for 25 min [5], the supernatant was transferred into another tube; chilled ethanol was added in which the ratio of ethanol to the sample was 3:1 $(v / \mathrm{v})$. The tube was mixed well and set at $4{ }^{\circ} \mathrm{C}$. The following day, the mixture was centrifuged at the conditions as described above, and the pellet was collected as EPS. Both EPS and slime track samples were dried at $60{ }^{\circ} \mathrm{C}$, and this material served as dry crude EPS. The crude EPS was then dissolved in $10 \%(w / v)$ trichloroacetic acid to remove proteins [5]. The supernatant was precipitated with chilled ethanol and centrifuged at the conditions described above. The pellet, referred to as partially purified EPS, was dried at $60{ }^{\circ} \mathrm{C}$ and stored at $4{ }^{\circ} \mathrm{C}$. Partially purified EPS was used for activity assessment and structural analysis.

\section{Chemical analysis}

The total carbohydrate and protein content of the slime track and crude EPS samples were analyzed by using the phenol sulphuric acid method [36] and the Bradford method [37], respectively. The sulfate group content was analyzed with the barium chloride gelatin method [38].

\section{Monosaccharide composition analysis by TLC}

Ten $\mathrm{mg}$ of partially purified EPSs was hydrolyzed in $1.0 \mathrm{~mL} 3 \mathrm{M}$ triflouroacetic acid $\left(100{ }^{\circ} \mathrm{C}, 8 \mathrm{~h}\right)$. After hydrolysis, TFA in the sample was removed by decompression evaporation. The hydrolyzed EPSs were redissolved in ultra-pure water. The supernatant was obtained by centrifugation at $13000 \mathrm{rpm}$ for $20 \mathrm{~min}$.

The hydrolyses were applied to silica gel plates using a developing solvent of butanol: acetone:pyridine: $\mathrm{H}_{2} \mathrm{O}$ [10:10:5:5 ( $\mathrm{v} / \mathrm{v} / \mathrm{v} / \mathrm{v})]$. Galactose, glucose and rhamnose were used as the standards. After TLC plate development, carbohydrate was visualized by spraying TLC plates with $1 \%$ aniline: $1 \%$ diphenylamine:85\% $\quad \mathrm{H}_{3} \mathrm{PO}_{4}$ [5:5:1 $(\mathrm{v} / \mathrm{v} / \mathrm{v})]$ and heating at $100{ }^{\circ} \mathrm{C}$ for $5 \mathrm{~min}$ to reveal the colored spots [39].

\section{Quantification of monomers by GC}

Samples were prepared according to the Kakasy method [40] with some modifications. The hydrolyses were dissolved in pyridine containing $2.5 \%$ hydroxylamine hydrochloride; after inositol (as an internal reference) was added to the solution, it was allowed to react at $80{ }^{\circ} \mathrm{C}$ for $30 \mathrm{~min}$ and cooled down to room temperature. Hexamethyldisilazane (HMDS) and TFA were then added, the mixture was allowed to react for further $30 \mathrm{~min}$ at $45{ }^{\circ} \mathrm{C}$ and cooled down again. One $\mathrm{mL}$ of silylate derivative was subjected to a DB-1 column $(30 \mathrm{~m} \times 0.35 \mu \mathrm{m} \times 0.25 \mu \mathrm{m})$ of GC (Aligent $6890 \mathrm{~N}$ ) fitted with a flame ionization detector
(FID). The operating conditions were as follow: the $\mathrm{N}_{2}$ carrier gas rate was $1.0 \mathrm{~mL} / \mathrm{min}$; injection and detector temperatures were $280{ }^{\circ} \mathrm{C}$ and $300{ }^{\circ} \mathrm{C}$, respectively; the column temperature was started at $60{ }^{\circ} \mathrm{C}$ for $1 \mathrm{~min}$, then increased to $210{ }^{\circ} \mathrm{C}$ at the rate of $20{ }^{\circ} \mathrm{C} / \mathrm{min}$ and maintained there for $5 \mathrm{~min}$, and finally increased to $300{ }^{\circ} \mathrm{C}$ at the rate of $100{ }^{\circ} \mathrm{C} /$ min and maintained there for $10 \mathrm{~min}$. Standard sugars (galactose, glucose, lactose, rhamnose and sucrose) with inositol as the internal standard were prepared and subjected to GC analysis separately in the same way.

\section{Antimicrobial activity \\ Well diffusion method}

Antimicrobial activity of the EPS and slime track samples was determined using the agar well diffusion method [4]. A volume of $100 \mathrm{~mL}$ of the cell suspension of the pathogenic culture $\left(10^{8} \mathrm{CFU} / \mathrm{mL}\right)$ was spread on the surface of a LB/Sabouraud dextrose agar plate using a sterile cotton swab. An amount of $100 \mu \mathrm{L}$ of the sample $(5 \mathrm{mg} / \mathrm{mL})$ was introduced into each well $(8 \mathrm{~mm}$ in diameter) in the plate. The positive antibacterial control used was erythromycin $(1.0 \mathrm{mg} / \mathrm{mL})$ and the antifungal control was ketoconazole $(1.0 \mathrm{mg} / \mathrm{mL})$. Sterile distilled water was used as the negative control. The plates were incubated for $37{ }^{\circ} \mathrm{C}$ in $8 \mathrm{~h}$. Antimicrobial activity was determined by measuring the diameter of the clear inhibition zone around each well.

\section{Minimum inhibitory concentration (MIC)}

MIC is the lowest concentration of an agent that inhibits the visible growth of a microorganism after overnight incubation [41]. MIC was carried out on the microorganisms that showed sensitivity to the samples and was done using the broth dilution method. MIC values were determined according Sen and Batra [42] with some modification. EPS/slime track samples were prepared with the concentration range of 0 to $20,480 \mu \mathrm{g} / \mathrm{mL}$. One $\mathrm{mL}$ of sterile culture medium was placed in a sterile test tube containing $100 \mu \mathrm{l}$ of microorganism suspension $\left(10^{5} \mathrm{CFU} / \mathrm{mL}\right)$. Then, $1.0 \mathrm{ml}$ of the EPS extract with a certain concentration was added to the mixture and incubated at $37{ }^{\circ} \mathrm{C}$ for $24 \mathrm{~h}$. After that, turbidity of the mixture was measured by using a spectrophotometer at a wavelength of $600, \mathrm{OD}_{600}$ value less than 0.01 was recorded as the MIC. Minimum bactericidal concentration $(\mathrm{MBC})$ and minimum fungicidal concentration (MFC) were defined as the lowest concentration of extract which showed no evidence of microbial growth on nutrient agar plates. MBC and MFC were investigated to confirm the MIC results.

The radical scavenging activity of slime track/EPS samples was measured with the use of the DPPH assay described by Monaki et al. [43] with some modifications. 
In brief, $80 \mu \mathrm{L}$ of the sample with a certain concentration ranging from 0 to $6.0 \mathrm{mg} / \mathrm{mL}$ was added to $120 \mu \mathrm{l}$ of $0.02 \mathrm{mg} / \mathrm{mL}$ DPPH prepared in a methanol solution. The mixture was mixed gently and incubated at room teperature for $30 \mathrm{~min}$ in the dark. Then, the absorbance was measured at $517 \mathrm{~nm}$ and the inhibition was calculated using the following formula

$$
\text { Scavenging rate }(\%)=\left[\left(\mathrm{A}_{0}-\mathrm{A}_{1}\right) / \mathrm{A}_{0}\right] \times 100
$$

where $A_{1}$ was absorbance of the sample and $A_{0}$ was the absorbance of the control [44]. The antioxidant ability of the sample was expressed as an IC50 value, which was defined as the concentration of sample that inhibits the formation of the DPPH radical by $50 \%$. An equal amount of methanol was added to the negative control, and ascorbic acid was used as the positive control.

\section{Anticancer activity}

The cytotoxicity of isolated EPS was determined using a sulforhodamine-B (SRB) assay [45]. The cancer cell lines $\left(10^{5}\right.$ cells $\left./ \mathrm{mL}\right)$ were seeded in a 96 -well microtiter plate and cultivated under standard conditions $\left(5 \% \mathrm{CO}_{2}\right.$ at $37^{\circ}$ C). Stock solution of the EPS samples were prepared in distilled water and serially diluted with sterile medium to obtain the desired concentrations. One hundred $\mu \mathrm{L}$ of the sample was then added to each well and incubated for another $48 \mathrm{~h}$ for cell attachment. Cells were fixed by gently layering of cold $50 \%$ TCA and incubated at $4{ }^{\circ} \mathrm{C}$ for $1 \mathrm{~h}$. The plate was then washed five times with distilled water and air-dried for $12 \mathrm{~h}$ at room temperature to avoid cell monolayer detachment. Cells were stained al least 15 min with $0.2 \%$ SRB dissolved in $1.0 \%$ acetic acid and subsequently washed 5 times with $1.0 \%$ acetic acid to remove unbound proteins. The plate was airdried. A tris-base solution was added to the wells to solubilize the dye. The plates were shaken gently for $10 \mathrm{~min}$ on a mechanical shaker. Distilled water and camptothecin $(0.01 \mu \mathrm{g} / \mathrm{mL})$ were used as negative and positive controls, respectively. A blank contained culture medium without cells. The optical density (OD) of the plate wells was recorded using a microplate reader at $560 \mathrm{~nm}$. Growth inhibition was calculated as.

$$
\% I=\left(1-\frac{\mathrm{A}}{\mathrm{B}}\right) \times 100 \%
$$

where A and B represent the absorbance of the test sample and the control [45].

\section{Statistical analysis}

All experiments were done in triplicate and all data are expressed as mean \pm standard eviation.

\section{Abbreviations}

CFU: Colony forming unit; DPPH: 2,2-diphenyl-1-picrylhydrazyl;

EPS: Exopolysaccharides; GC: Gas chromatography; IC50: Half inhibitory concentration; MBC: Minimum bactericidal concentration; MFC: Minimum fungicidal concentration; MIC: Minimum inhibitory concentration; TCA: Trichloroacetic acid; TFA: Triflouroacetic acid; TLC: Thin layer chromatography

\section{Acknowledgements}

We would like to thank the Vietnam National Foundation for Science and Technology Development (NAFOSTED for funding this work.

\section{Funding}

This research was funded by the Vietnam National Foundation for Science and Technology Development (NAFOSTED) under grant number 106-NN.04-2015.16.

\section{Availability of data and materials}

The datasets used and/or analysed during the current study available from the corresponding author on reasonable request.

\section{Authors' contributions}

TH carried out the experiments and data analysis, TP handled the GC analysis, HT helped with the culture techniques and the manuscript preparation, and SS edited the manuscript prior to submission. All the authors read and approved the manuscript for submission.

\section{Competing interest}

The authors declare that they have no competing interests with respect to any aspect of this manuscript or the project reported in the manuscript.

Ethics approval and consent to participate

Not applicable.

Consent for publication

Not applicable.

\section{Publisher's Note}

Springer Nature remains neutral with regard to jurisdictional claims in published maps and institutional affiliations.

\section{Author details}

${ }^{1}$ School of Biotechnology, International University, VNU-HCM, Block 6, LinhTrung Ward, Thu Duc District, Ho Chi Minh City 70000, Vietnam. ${ }^{2}$ Institute of Chemical Technology, Vietnam Academy of Science and Technology, 01-Mac Dinh Chi Street, District 1, Ho Chi Minh City 70000, Vietnam. ${ }^{3}$ Department of Biological Sciences, University of Arkansas, Fayetteville, AR 72701, USA.

Received: 24 May 2017 Accepted: 31 October 2017

Published online: 09 November 2017

\section{References}

1. Nwodo UU, Green E, Okoh Al. Bacterial exopolysaccharides: functionality and prospects. Int J Mol Sci. 2012;13:14002-15.

2. Singha TK. Microbial extracellular polymeric substances: production, isolation and applications. IOSR J Pharm. 2012:2:276-81.

3. Orsod M, Joseph M, Huyop F. Characterization of exopolysaccharides produced by Bacillus cereus and Brachybacterium sp. isolated from Asian Sea bass (Lates calcarifer). Malays. J Microbiol. 2012;8:170-4.

4. Mahendran S, Saravanan S, Vijayabaskar P, Anandapandian KTK, Shankar T. Antibacterial potential of microbial exopolysaccharide from Ganoderma lucidium and Lysinibacillus fusiformis. Int J Recent Sci Res. 2013;4:501-5.

5. Osama H, El S, El Kader A, El-Sayed M, Salem HM, Manal GM, Asker MS, Saher SM. Isolation, characterization and biological activities of exopolysaccharide produced by Bacillus marinus. Der Pharma Chem. 2015;7:200-8.

6. Thetsrimuang C, Khammuang S, Chiablaem K, Srisomsap C, Sarnthima R. Antioxidant properties and cytotoxicity of crude polysaccharides from Lentinus polychrous Lév. Food Chem. 2011;128:634-9. 
7. Sun X, Hao L, Ma H, Li T, Zheng L, Ma Z, Zhai G, Wang L, Gao S, Liu X, Jia M, Jia M. Extraction and in vitro antioxidant activity of exopolysaccharide by Pleurotus eryngii SI-02. Braz J Microbiol. 2013;44:1081-8.

8. Sharma SK. Optimized extraction and antioxidant activities of polysaccharides from two entomogenous fungi. J Bioanal Biomed. 2015;7:180-7.

9. Wang K, Li W, Rui X, Chen X, Jiang M, Dong M. Structural characterization and bioactivity of released exopolysaccharides from Lactobacillus plantarum 70810. Int J Biol Macromol. 2014;67:71-8.

10. Li H, Liu X, Xu Y, Wang X, Zhu H. Structure and antitumor activity of the extracellular polysaccharides from Aspergillus aculeatus via apoptosis and cell cycle arrest. Glycoconj J. 2016;33:975-84.

11. Stephenson SL, Stempen H. Myxomycetes: a handbook of slime molds. Oregon: Timber Press; 1994.

12. Dembitsky VM, Řezanka T, Spižek J, Hanuš LO. Secondary metabolites of slime molds. Phytochemistry. 2005;66(7):747-69.

13. Misono $Y$, Ishibashi $M$, Ito A. Bahiensol, a new glycerolipid from a cultured myxomycete Didymium bahiense var. bahiense. Chem Pharm Bull. 2003;51(5):612-3.

14. Herrera NA, Rojas C, Franco-Molano AE, Stephenson SL, Echeverri F. Physarella oblonga centered bioassays for testing the biological activity of myxomycetes. Mycosphere. 2011;2(6):637-44.

15. Murakami-Murofushi K, Uchiyama A, Fujiwara Y, Kobayashi T, Kobayashi S, Mukai M, Murofushi H, Tigyi G. Biological functions of a novel lipid mediator, cyclic phosphatidic acid. Biochim Biophys Acta Mol Cell Biol Lipids. 2002;1582(1):1-7.

16. Hosoya T, Yamamoto Y, Uehara Y, Hayashi M, Komiyama K, Ishibashi M. New cytotoxic bisindole alkaloids with protein tyrosine kinase inhibitory activity from a myxomycete Lycogala epidendrum. Bioorg Med Chem Lett. 2005; 15(11):2776-80.

17. Kaniwa K, Ohtsuki T, Yamamoto Y, Ishibashi M, Kehokorins A-C. Novel cytotoxic dibenzofurans isolated from the myxomycete Trichia favoginea Var. persimilis. Tetrahedron Lett. 2006;47(10):1505-8.

18. Clark J, Haskins EF. Myxomycete plasmodial biology: a review. Mycosphere. 2015;6:643-58

19. McCormick JJ, Blomquis JC, Rusch HP. Isolation and characterization of an extracellular polysaccharide from Physarum polycephalum. J Bacteriol. 1970;104:1110-8.

20. Simon HL, Henney HR. Chemical composition of slime from three species of myxomycetes. FEBS Lett. 1970;7:80-2.

21. Sperl TG. Isolation and characterization of the Physarum polycephalum extracellular polysaccharides. Food Biotechnol. 1990;4(2):663-8.

22. Asgari M, Henney HR Jr. Inhibition of growth and cell wall morphogenesis of Bacillus subtilis by extracellular slime produced by Physarum flavicomum. Cytobios. 1977;20:163-77.

23. Henney JHR, Henney MR. Nutritional requirements for the growth in pure culture of the myxomycete Physarum rigidum and related species. Microbiology. 1968;53(3):333-9.

24. Farr D, Amster H, Horisberger M. Composition and partial structure of the extracellular polysaccharide of Physarum polycephalum. Carbohydr Res. 1972;24:207-9.

25. Gamar-Nourani L, Blondeau K, Simonet JM. Influence of culture conditions on exopolysaccharide production by Lactobacillus rhamnosus strain C83. J Appl Microbiol. 1998;85:664-72.

26. Degeest B, Vaningelgem F, Vuyst LD. Microbial physiology, fermentation kinetics, and process engineering of heteropolysaccharide production by lactic acid bacteria. Int Dairy J. 2011;11:747-57.

27. Li S, Huang R, Shah NP, Tao X, Xiong Y, Wei H. Antioxidant and antibacterial activities of exopolysaccharides from Bifidobacterium bifidum WBIN03 and Lactobacillus plantarum R315. J Dairy Sci. 2014;97:7334-43.

28. Kiran GS, Priyadharshini S, Anitha K, Gnanamani E, Selvin J. Characterization of an exopolysaccharide from probiont Enterobacter faecalis MSI12 and its effect on the disruption of Candida albicans biofilm. RSC Adv. 2015;5:71573-85.

29. Olugbami JO, Gbadegesin MA, Odunola OA. In vitro free radical scavenging and antioxidant properties of ethanol extract of Terminalia glaucescens. Pharmacognosy Res. 2015;7:49-56.

30. Vamanu E. Biological activities of the polysaccharides produced in submerged culture of two edible Pleurotus ostreatus mushrooms. Biomed Res Int. 2012;2012(565974):8.

31. He P, Geng L, Mao D, Production XC. Characterization and antioxidant activity of exopolysaccharides from submerged culture of Morchella crassipes. Bioprocess Biosyst Eng. 2012;35:1325-32.
32. Huang QL, Siu KC, Wang WQ, Cheung YC, Fractionation WJY. Characterization and antioxidant activity of exopolysaccharides from fermentation broth of a Cordyceps sinensis fungus. Process Biochem. 2013;48:380-6.

33. Tran HTM, Stephenson SL, Chen Z, Pollock ED, Goggin FL. Evaluating the potential use of myxomycetes as a source of lipids for biodiesel production. Bioresour Technol. 2012;123:386-9.

34. Tran H, Stephenson S, Pollock E. Evaluation of Physarum polycephalum plasmodial growth and lipid production using rice bran as a carbon source. BMC Biotechnol. 2015;15:67.

35. Dubois M, Gilles KA, Hamilton JK, Rebers PA, Smith F. Colorimetric method for determination of sugars and related substances. Anal Chem. 1956;28:350-6.

36. Bradford MM. A rapid and sensitive method for the quantitation of microgram quantities of protein utilizing the principle of protein-dye binding. Anal Biochem. 1976;72:248-54.

37. Dodgson KS, Price RG. A note on the determination of the ester sulphate content of sulphated polysaccharides. Biochem J. 1962;84:106-10.

38. Kumar CSC, Chandraju S, Mythil R, Ahmad T, Gowda NM. Extraction of sugars from black gram peels by reversed-phase liquid chromatography systems and identification by TLC and mass analysis. Adv Anal Chem. 2012;2:32-6.

39. Kakasy A, Füzfai Z, Kursinszki L, Molnár-Perl I, Lemberkovics É. Analysis of non-volatile constituents in Dracocephalum species by HPLC and GC-MS. Chromatographia. 2006;63(13):17-22.

40. Wiegand I, Hilper K, Hancock RE. Agar and broth dilution methods to determine the minimal inhibitory concentration (MIC) of antimicrobial substances. Nat Protoc. 2008;3:163-75.

41. Sen A, Batra A. Evaluation of antimicrobial activity of different solvent extracts of medicinal plant: Melia azedarach L. Int J Curr Pharm Res. 2012;4:67-73.

42. Osińska-Jaroszuk M, Jaszek M, Mizerska-Dudk M, Błachowicz A, Rejczak TP, Janusz G, Wydrych J, Polak J, Jarosz-Wilkołazka A, Kandefer-Szerszeń M. Exopolysaccharide from Ganoderma applanatum as a promising bioactive compound with cytostatic and antibacterial properties. Biomed Res Int. 2014;2014(743812):10,

43. Ma YP, Mao DB, Geng $\sqcup$, Zhang WY, Wang Z, Xu CP. Production optimization, molecular characterization and biological activities of exopolysaccharides from Xylaria nigripes. Chem Biochem Eng Q. 2013;27:177-84.

44. Akindele AJ, Wani ZA, Sharma S, Mahajan G, Satt NK, Adeyemi OO, Mondhe DM, Saxena AK. Vitro and in vivo anticancer activity of root extracts of Sansevieria liberica Gerome and Labroy (Agavaceae). Evid Based Complement Alternat Med. 2015;2015:560404.

45. Vichai V, Kirtikara K, Sulforhodamine B. Colorimetric assay for cytotoxicity screening. Nat Protoc. 2006;1:1112-6.

\section{Submit your next manuscript to BioMed Central and we will help you at every step:}

- We accept pre-submission inquiries

- Our selector tool helps you to find the most relevant journal

- We provide round the clock customer support

- Convenient online submission

- Thorough peer review

- Inclusion in PubMed and all major indexing services

- Maximum visibility for your research

Submit your manuscript at www.biomedcentral.com/submit
C) Biomed Central 